\title{
A Magnetically Actuated Resonant Mass Sensor With Integrated Optical Readout
}

\author{
Alibey Ozturk, H. Ilker Ocakli, Natali Ozber, Hakan Urey, Member, IEEE, I. Halil Kavakli, and \\ B. Erdem Alaca, Member, IEEE
}

\begin{abstract}
Nickel cantilevers with integrated diffraction gratings are used as resonant mass sensors with a resolution of 500 femtograms. Their applicability to biosensing is demonstrated with human opioid receptors. The device is fabricated through a single-mask lithographic process. The microoptical readout provides a simple measurement platform with one external photodiode. Thanks to its ac operation principle, the device is immune to environmental noise and entails a high tolerance to fabrication defects. Obtained signal-to-noise ratio is comparable to that of a high-end Doppler vibrometer. The device with these aspects for systems integration and microarray technology is a candidate for low-cost portable sensors.
\end{abstract}

Index Terms-Interferometry, magnetic films, microelectromechanical devices, resonators.

\section{INTRODUCTION}

G ROWING demand for methods of detecting biochemical species lead to a great interest in resonant micro-, and nanoelectromechanical systems [1], [2]. Utilizing state-of-the-art fabrication techniques, it became possible to obtain inexpensive sensors with a very high resolution [3], [4]. The majority of the reported studies are based on various cantilever designs. The resonance frequency $\omega_{0}$ of a cantilever is described through the following formula:

$$
\omega_{0}=\sqrt{\frac{k}{m}}
$$

where $k$ is the stiffness and $m$ is the effective suspended mass of the cantilever. Assuming that the stiffness of the cantilever is unchanged after the "loading," the accumulated mass can be calculated using the shift in the resonance frequency.

Sensitivity of the cantilever transducers can be defined in various ways. One of the most frequently employed definitions for

Manuscript received May 20, 2008; revised July 23, 2008. First published September 3, 2008; current version published November 12, 2008. This work was supported by TÜBITAK under Grant 105E148. The work of H. Urey and I. H. Kavakli was supported by a TÜBA-GEBIP Distinguished Young Scientist award.

The authors are with the College of Engineering, Koc University, Sariyer 34450, Istanbul, Turkey (e-mail: hurey@ku.edu.tr; hkavakli@ku.edu.tr; ealaca@ku.edu.tr).

Color versions of one or more of the figures in this letter are available online at http://ieeexplore.ieee.org.

Digital Object Identifier 10.1109/LPT.2008.2005253 sensitivity is given in terms of the mass responsivity $\Re$ [4], relating the minimum detectable mass by the sensor $\delta m$ to the frequency shift $\delta \omega_{0}$

$$
\Re=\frac{\partial \omega_{0}}{\partial m}=\frac{-\omega_{0}}{2 m} .
$$

Based on the linearization of the mass and frequency relation, the above formulation is valid for small additions of mass. Nevertheless, it highlights the necessity for miniaturization for increased sensitivity accompanied by an increase in $\omega_{0}$ and a decrease in $m$.

The major purpose of this study is to demonstrate the same transduction principle with an integrated optical readout and magnetic cantilevers. The fabrication sequence is entirely based on forming of the resonating structure and the diffraction grating by electroplating of $\mathrm{Ni}$, which also brings about the possibility of implementing magnetic actuation and operation in fluids. The silicon substrate lying beneath the released cantilevers serves as the reference surface for the interferometric detection of the cantilever motion. The typical surface roughness associated with the electroplating of $\mathrm{Ni}$ as the moving reflector and $\mathrm{KOH}$ etching of $\mathrm{Si}$ as the reference is observed to be adequate for a reliable interferometric measurement adding up to the simplicity of the proposed fabrication method. In the following, the fabrication will be explained first, followed by a discussion of the verification of the measurements against the readings of a laser Doppler vibrometer (LDV). The mass sensitivity of the device is then calculated using controlled deposition of Au layers. Finally, the applicability of the device as a biosensor is demonstrated with human opioid receptor proteins.

\section{EXPERIMENTAL DEMONSTRATION}

\section{A. Fabrication and Setup}

The fabrication sequence of the proposed device is given in Fig. 1(a). After standard cleaning of a $\langle 100\rangle$ 4-in single-crystal silicon wafer with a thickness of $525 \pm 25 \mu \mathrm{m}$ and a resistivity of $0.1-0.5 \Omega \cdot \mathrm{cm}$, a $10 \mathrm{~nm} / 100 \mathrm{~nm} \mathrm{Cr}-\mathrm{Au}$ layer was sputtered. Au layer served both as the seed layer for the subsequent electroplating step and as the functionalization surface for the biomolecules. Ultraviolet (UV) photolithography was then employed to pattern the Au surface. The gratings were tilted with respect to the long axis of the cantilever to avoid the diffracted light from the horizontal and vertical edges of the structures. 


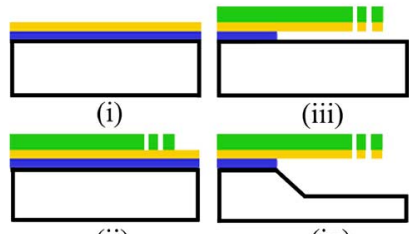

(ii)

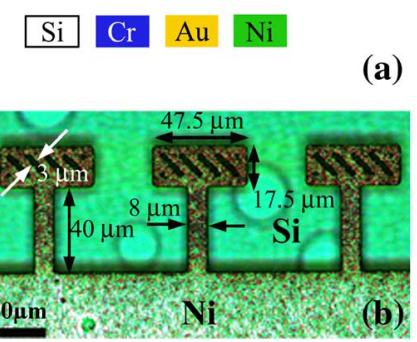

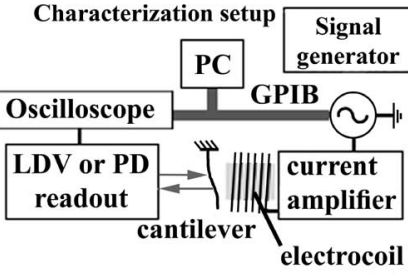

(c)

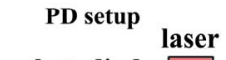

photodiode $\square$

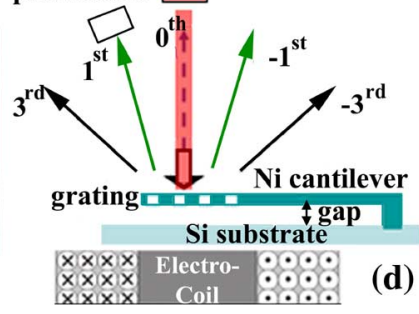

Fig. 1. (a) Fabrication flow: (i) Sputtering of $\mathrm{Cr}(10 \mathrm{~nm})$ and $\mathrm{Au}(100 \mathrm{~nm})$; (ii) formation of the structural layer (cantilevers and gratings) via UV photolithography and electroplating; (iii) $\mathrm{Au}$ and $\mathrm{Cr}$ etch; (iv) Release of the $\mathrm{Ni}$ structures with $\mathrm{KOH}$ etch. (b) An array of fabricated cantilevers with a thickness of $0.7 \mu \mathrm{m}$ and with a platform of four $6-\mu \mathrm{m}$-period diffraction gratings. (c) Characterization setup. (d) Optical readout and magnetic actuation principle. PD intensity is a sinusoidal function of the gap with a period of $\lambda / 2$.

After the lithography, the wafer was diced and the cantilevers were obtained by electroplating of $\mathrm{Ni}$ on individual dice in a nickel sulphamate bath. The thickness and the roughness of the cantilevers were controlled by arranging the current density, the temperature and the duration of the deposition. For current densities of 5 to $10 \mathrm{~mA} / \mathrm{cm}^{2}$, the average roughness achieved was less than $20 \mathrm{~nm}$, which is generally acceptable for optical studies. With increasing current density, the roughness was also observed to increase. To establish a uniform deposition, each of the four corners of the die was used as the electrical contact and the bath was continuously stirred. During the electroplating, the temperature was set to $40{ }^{\circ} \mathrm{C}$ and current densities varying from 5 to $25 \mathrm{~mA} / \mathrm{cm}^{2}$ were used. Following the formation of the cantilevers, photoresist was stripped off and the seed layer was etched. Etching of Au was critical since it had to be preserved underneath the cantilevers for further functionalization. Finally, the cantilevers were released by anisotropic $\mathrm{KOH}$ etching carried out at $80^{\circ} \mathrm{C}$ and in a $35 \%$ concentrated solution to minimize the roughness of the Si surface. Ni served as a natural etch mask during this step eliminating the need for further lithography. $\mathrm{Ni}$ also imparts corrosion resistance, a necessary feature for reliability in biosensor applications. Fig. 1(b) shows an array of cantilevers ready for actuation, where the roughness of both $\mathrm{Ni}$ and Si surfaces is evident.

The actuation of the cantilevers was carried out by means of an external electromagnetic coil with the prospect of being micromachined as a part of the process [5]. Magnetic actuation eliminates the drawbacks such as charging, stiction due to small gaps and other limitations associated with the electrostatic method and also enables operation in liquid media. The cantilevers were excited with a signal generator followed by a current amplifier. For the characterization of the resonant behavior both a commercial LDV (Polytec OFV-2500 with a bandwidth of $0.5 \mathrm{~Hz}-250 \mathrm{kHz}$ ) and a photodiode (PD) circuit utilizing the grating interferometry were used [Fig. 1(c)]. The shift in the resonance frequency was employed to monitor the change of mass of the structure. LDV served as a measure of comparison and verification of $\mathrm{PD}$ measurements. The PD setup consisted of a laser source and a PD with transimpedance amplifier. No vibration isolation was employed for PD measurements, whereas the optical table needed floating for LDV readings.

The grating interferometry principle is depicted in Fig. 1(d). A deflection of the cantilever causes modulation in the zeroth and first orders of the diffracted light. The intensity changes periodically with the air gap between the cantilever and the substrate with a period of $\lambda / 2$, where $\lambda$ is the wavelength of the laser. Thus, the deflection can be measured by monitoring the sinusoidal intensity modulation. An unambiguous detection range can be increased substantially by using two lasers and two detectors for the readout [6]. In order to extract the frequency response and the resonance frequency of the beams, the driving signal was swept over a band of frequencies and the PD output is recorded. The resonance frequency can be detected by recording the frequency where the maximum peak-to-peak voltage is observed [5]-[7]. With appropriate circuitry, the PD output signal can be used to keep the structure at resonance as well. Shot-noise-limited detection can be achieved by monitoring both zeroth and first order diffracted light to cancel laser and other noise sources [7].

\section{B. Measurements}

In order to compare the resonance frequencies measured by the LDV and PD setups mentioned above, cantilevers with various lengths were used. The thickness was kept constant at $0.7 \mu \mathrm{m}$ and the width was $8 \mu \mathrm{m}$. Resonance frequencies ranging from 18 to $160 \mathrm{kHz}$ were measured. PD measurements were observed to be in agreement with LDV readings. A comparison for a specific cantilever is given in Fig. 2 and shows that the noise level in the PD measurement is comparable to that of LDV with a root-mean-square (rms) error of $1.5 \mathrm{~Hz}$ around resonance as extracted from the phase diagram in Fig. 2(b). Here the values of deflection were measured by LDV in nanometers and PD readings were fit to these data for scaling purposes.

After the verification of the resonance measurements, the operation and the mass responsivity of the fabricated devices were studied with two different methods. In the first method, a well calibrated sputtering system was utilized to deposit known amounts of gold on the sensor and the associated shift in the resonance frequency was measured. The measurements were also validated with finite-element numerical analysis. Au was deposited in four discrete steps. The associated frequency measurements are given in Fig. 3. The specific cantilever used for the measurement had a width of $6 \mu \mathrm{m}$ and a length of $60 \mu \mathrm{m}$. Its thickness was measured to be $1.4 \mu \mathrm{m}$. In the first two sessions of loading, a longer deposition was carried out leading to an accumulation of 173.0 and $189.0 \mathrm{pg}$ of $\mathrm{Au}$. In the next two sessions the deposition time was reduced and the measured mass accumulation was found to be 80.0 and $102.5 \mathrm{pg}$. According to these results, the inverse mass responsivity of the particular device is $0.32 \mathrm{pg} \cdot \mathrm{Hz}^{-1}$. Combined with the already measured rms error of the frequency measurements this 

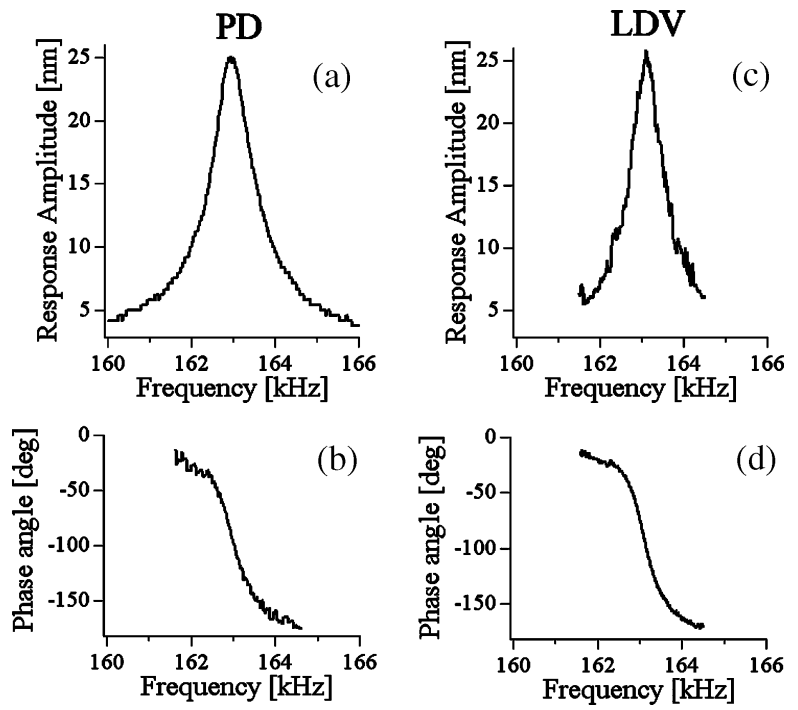

Fig. 2. Comparison of LDV and PD measurements for a cantilever of $8 \mu \mathrm{m} \times$ $40 \mu \mathrm{m}$. (a) PD resonance curve. (b) PD phase shift. (c) LDV resonance curve. (d) LDV phase shift.

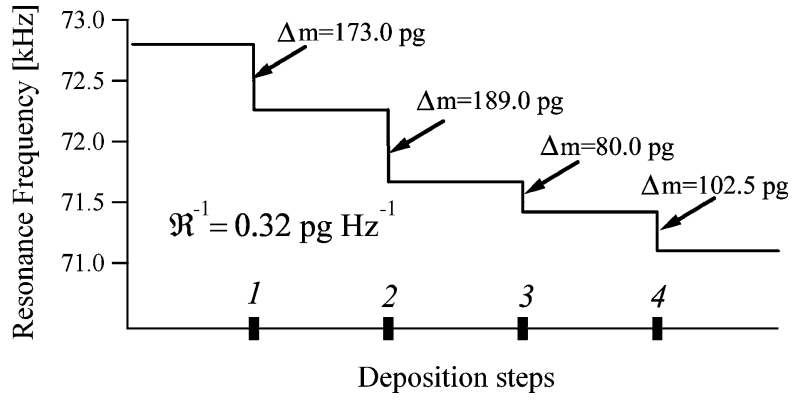

Fig. 3. Change in the resonance frequency of a cantilever due to a sequence of controlled Au deposition.

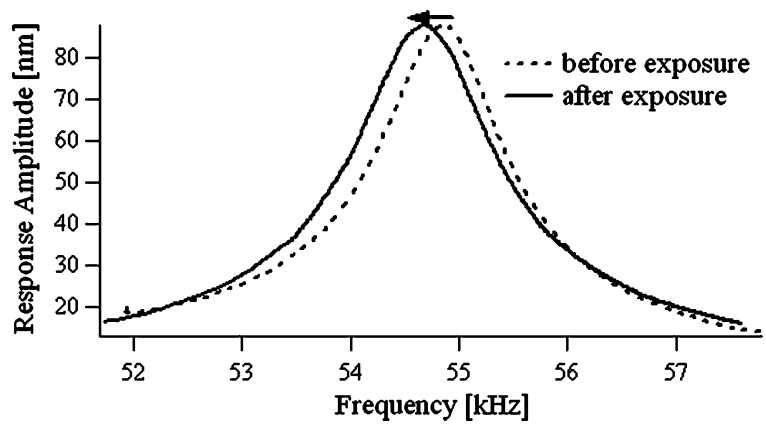

Fig. 4. Resonance frequency shift of a cantilever due the attachment of human opioid receptors to the Au surface.

leads to a mass resolution of $0.5 \mathrm{pg}$ at $72 \mathrm{kHz}$. Increasing the PD signal either by using an external permanent magnet or by increasing the driving current of the coil would further enhance the resolution.

In the second method, protein molecules were attached on a cantilever of dimensions $7 \mu \mathrm{m} \times 70 \mu \mathrm{m}$ and the resonance frequency shift was measured. The thickness of the cantilever in this case was $1.3 \mu \mathrm{m}$. For this purpose the cDNA of human kappa opioid receptor (hKOR) was PCR amplified and cloned into pMAL-c2x vector and named as pNO1. pNO1 was transferred into E.coli and hKOR protein was expressed with IPTG for $9 \mathrm{~h}$ at $37^{\circ} \mathrm{C}$. The receptor was then purified through Ni-NTA agarose resin. Then it was immobilized on the Au surface of the cantilever via thiol groups of DSP linker through His tag [8]. The shift of $180 \mathrm{~Hz}$ shown in Fig. 4 corresponds to a mass accumulation of $85 \mathrm{pg}$. The formation of a monolayer of proteins on the cantilever surface is estimated to lead to a mass accumulation of $40 \mathrm{pg}$. However, since hKOR is a membrane protein, it is possible that it forms irregular micelles during the functionalization of the gold surface.

\section{CONCLUSION}

The resonant behavior of Ni cantilevers was observed with the help of integrated diffraction gratings. A resolution of $0.5 \mathrm{pg}$ was achieved using $72-\mathrm{kHz}$ resonant devices operating at ambient pressure and the applicability as a biosensor was demonstrated. Thanks to the noise rejection capability of ac detection and the simple grating readout principle, the signal-to-noise ratio of the optical readout method was shown to be comparable to that of LDV measurements even when the substrate and the nickel surfaces are rough due to various etching and deposition steps. Furthermore, the integrated readout did not require vibration isolation whereas LDV measurements did. Increased tolerance to fabrication defects and noise has certain implications in the simplicity and yield of the fabrication technique.

The system also has the capability for parallel array readout with a single PD, where multiple beams with slightly different resonance frequencies are monitored simultaneously, providing an important advantage over most cantilever-based sensors. Combined with the electromagnetic actuation, which, in principle, can be carried out on-chip, the observations indicate the potential for a system-level integration for realizing a low-cost, portable and disposable sensor with a low-noise readout.

\section{REFERENCES}

[1] C. Ziegler, "Cantilever-based biosensors," Anal. Bioanal. Chem., vol. 379, pp. 946-959, 2004.

[2] K. K. Park, H. J. Lee, G. G. Yaralioglu, A. S. Ergun, Ö. Oralkan, M Kupnik, C. F. Quate, B. T. Khuri-Yakub, T. Braun, J.-P. Ramseyer, H. P. Lang, M. Hegner, C. Gerber, and J. K. Gimzewski, "Capacitive micromachined ultrasonic transducers for chemical detection in nitrogen," Appl. Phys. Lett., vol. 91, no. 094102, 2007.

[3] A. Gupta, D. Akin, and R. Bashir, "Single virus particle mass detection using microresonators with nanoscale thickness," Appl. Phys. Lett., vol. 84, no. 11, pp. 1976-1978, 2004.

[4] K. L. Ekinci, X. M. H. Huang, and M. L. Roukes, "Ultrasensitive nanoelectromechanical mass detection," Appl. Phys. Lett., vol. 84, no. 22, pp. 4469-4471, 2004.

[5] S. O. Isikman, O. Ergeneman, A. D. Yalcinkaya, and H. Urey, "Modeling and characterization of soft magnetic film actuated 2-D scanners," IEEE J. Sel. Topics Quantum Electron., vol. 12, no. 2, pp. 283-289, Mar./Apr. 2007.

[6] O. Ferhanoglu, M. F. Toy, and H. Urey, "Two-wavelength grating interferometry for MEMS sensors," IEEE Photon. Technol. Lett., vol. 19, no. 23, pp. 1895-1897, Dec. 1, 2007.

[7] N. A. Hall and F. L. Degertekin, "Integrated optical interferometric detection method for micromachined capacitive acoustic transducers," Appl. Phys. Lett., vol. 80, no. 20, pp. 3859-3861, 2002.

[8] S. A. Trammell, L. Wang, J. M. Zullo, R. Shashidhar, and N. Lebedev, "Orientated binding of photosynthetic reaction centers on gold using Ni-NTS self-assembled monolayers," Biosens. Bioelectron., vol. 19, pp. 1649-1655, 2004. 